\title{
The host galaxy and optical light curve of the gamma-ray burst GRB 980703
}

\author{
S. Holland ${ }^{1,2}$, J. P. U. Fynbo ${ }^{3}$, J. Hjorth ${ }^{4}$, J. Gorosabel ${ }^{5}$, H. Pedersen ${ }^{4}$, M. I. Andersen ${ }^{6}$, A. Dar ${ }^{7}$, \\ B. Thomsen ${ }^{1}$, P. Møller ${ }^{3}$, G. Björnsson ${ }^{8}$, A. O. Jaunsen ${ }^{9}$, P. Natarajan ${ }^{10}$, and N. Tanvir ${ }^{11}$ \\ 1 Institute of Physics and Astronomy, University of Aarhus, 8000 Århus C., Denmark \\ e-mail: bt@ifa.au.dk \\ 2 Department of Physics, University of Notre Dame, Notre Dame, IN 46556-5670, USA \\ e-mail: sholland@nd.edu \\ 3 European Southern Observatory, Karl-Schwarzschild-Straße 2, 85748 Garching, Germany \\ e-mail: jfynbo@eso.org; pmoller@eso.org \\ 4 Astronomical Observatory, University of Copenhagen, Juliane Maries Vej 30, 2100 Copenhagen $\varnothing$, Denmark \\ e-mail: jens@astro.ku.dk; holger@astro.ku.dk \\ ${ }^{5}$ Danish Space Research Institute, Juliane Maries Vej 30, 2100 Copenhagen $\varnothing$, Denmark \\ e-mail: jgu@dsri.dk \\ ${ }^{6}$ Division of Astronomy, University of Oulu, PO Box 3000, 90014 Oulu, Finland \\ e-mail: manderse@sun3.oulu.fi \\ 7 Department of Physics, Technion, Haifa 32000, Israel \\ e-mail: arnon.dar@cern.ch \\ 8 Science Institute, Dunhagi 3, University of Iceland, 107 Reykjavik, Iceland \\ e-mail: gulli@raunvis.hi.is \\ 9 European Southern Observatory, Casilla 19001, Santiago 19, Chile \\ e-mail: ajaunsen@eso.org \\ 10 Department of Astronomy, Yale University, New Haven, CT 06520-8181, USA \\ e-mail: priya@astro.yale.edu \\ 11 Department of Physical Sciences, University of Hertfordshire, College Lane, Hatfield, Hertfordshire, \\ AL10 9AB, UK \\ e-mail: nrt@star.herts.ac.uk
}

Received 11 December 2000 / Accepted 27 February 2001

\begin{abstract}
We present deep HST/STIS and ground-based photometry of the host galaxy of the gamma-ray burst GRB 980703 taken 17, 551, 710, and 716 days after the burst. We find that the host is a blue, slightly overluminous galaxy with $V_{\text {gal }}=23.00 \pm 0.10,(V-R)_{\text {gal }}=0.43 \pm 0.13$, and a centre that is $\approx 0.2$ mag bluer than the outer regions of the galaxy. The galaxy has a star-formation rate of $8-13 \mathcal{M}_{\odot} \mathrm{yr}^{-1}$, assuming no extinction in the host. We find that the galaxy is best fit by a Sersic $R^{1 / n}$ profile with $n \approx 1.0$ and a half-light radius of $0^{\prime \prime} 13\left(=0.72 h_{100}^{-1}\right.$ proper $\left.\mathrm{kpc}\right)$. This corresponds to an exponential disk with a scale radius of $0^{\prime \prime} .22$ $\left(=1.21 h_{100}^{-1}\right.$ proper $\left.\mathrm{kpc}\right)$. Subtracting a fit with elliptical isophotes leaves large residuals, which suggests that the host galaxy has a somewhat irregular morphology, but we are unable to connect the location of GRB 980703 with any special features in the host. The host galaxy appears to be a typical example of a compact star forming galaxy similar to those found in the Hubble Deep Field North. The $R$-band light curve of the optical afterglow associated with this gamma-ray burst is consistent with a single power-law decay having a slope of $\alpha=-1.37 \pm 0.14$. Due to the bright underlying host galaxy the late time properties of the light-curve are very poorly constrained. The decay of the optical light curve is consistent with a contribution from an underlying type Ic supernova like SN1998bw, or a dust echo, but such contributions cannot be securely established.
\end{abstract}

Key words. supernovae: individual - galaxies: individual - galaxies: photometry - galaxies: structure - gamma rays: bursts

\footnotetext{
Send offprint requests to: S. Holland, e-mail: sholland@nd.edu

* Based on observations made with the NASA/ESA Hubble Space Telescope $(H S T)$, obtained at the Space Telescope Science Institute, which is operated by the Association of Universities for Research in Astronomy, Inc., under NASA contract NAS5-26555.
}

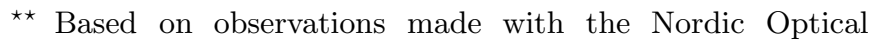
Telescope (NOT), operated on the island of La Palma jointly by Denmark, Finland, Iceland, Norway, and Sweden.

$\star \star \star$ Based on observations made at the 2.2-m telescope of the German-Spanish Calar Alto Observatory. 


\section{Introduction}

GRB 980703 (BATSE trigger 6891) was detected on 1998 July 3.182 UT by the All-Sky Monitor on the Rossi X-Ray Timing Explorer satellite (Levine et al. 1998). Frail et al. (1998) and Zapatero Osorio et al. (1998) identified a fading optical afterglow (OA), and Djorgovski et al. (1998b) derived a redshift of $z=0.9661 \pm 0.0001$ from absorption and emission lines in the combined spectrum of the OA and host galaxy. They also derived a star-formation rate of $\approx 10 \mathcal{M}_{\odot} \mathrm{yr}^{-1}$ for the host galaxy from the strength of the $[\mathrm{O}$ II] emission line. They stressed that the star formation rate depends on the adopted extinction and on the star-formation indicator used, but concluded that the host is a compact starburst galaxy. $R$-band optical light curves of the OA + host galaxy were presented by Bloom et al. (1998), Castro-Tirado et al. (1999), and Vreeswijk et al. (1999), who all found that the optical light decayed as a single power law with a slope of $\alpha \approx-1.4$, and all estimated the magnitude of the host galaxy to be $R_{\text {gal }} \approx 22.6$. Sokolov et al. (2001) used late-time photometry of the OA + host to estimate global star-formation rates in the host galaxy of $15-66 \mathcal{M}_{\odot} \mathrm{yr}^{-1}$, depending on the details of the initial mass function.

The host galaxy of GRB 980703 appears to have a high extinction along the line of sight to the gamma-ray burst (GRB). Castro-Tirado et al. (1999) found $A_{V}=2.2$ from the slope of $X$-ray/optical/infrared fluxes 0.9 days after the burst, Vreeswijk et al. (1999) used a similar method to find $A_{V}=1.50 \pm 0.111 .2$ days after the burst, Djorgovski et al. (1998a) found $A_{V} \approx 0.3 \pm 0.3$ from the Balmer decrement 4.4 days after the burst, and Bloom et al. (1998) estimated $A_{V}=0.9 \pm 0.25 .3$ days after the burst. The different (and formally inconsistent) values of $A_{V}$ may be due to different methods for measuring the extinction probing different regions of the host galaxy, or may indicate that the environment where the burst occurred is changing with time.

We have obtained new ground-based and Hubble Space Telescope (HST) Space Telescope Imaging Spectrograph (STIS) images of the host galaxy of GRB 980703 taken 17, 551, 710, and 716 days after the burst. These data are discussed in Sect. 2. The nature of the host galaxy and the location of the GRB within the galaxy are discussed in Sect. 3, and the light curve of the $\mathrm{OA}$ is discussed in Sect. 4. Unless otherwise noted we have assumed a cosmology where $H_{0}=100 h_{100} \mathrm{~km} \mathrm{~s}^{-1} \mathrm{Mpc}^{-1}, \Omega_{m}=0.3$, and $\Omega_{\Lambda}=0.7$. For this cosmology a redshift of 0.9661 corresponds to a luminosity distance of $4.43 h_{100}^{-1} \mathrm{Gpc}$ and a distance modulus of $43.23-5 \log _{10}\left(h_{100}\right)$. One arcsecond corresponds to $10.9 h_{100}^{-1}$ comoving kpc or $5.6 h_{100}^{-1}$ proper kpc, and the lookback time is $5.3 h_{100}^{-1}$ Gyr.

\section{Observations and data reductions}

\subsection{Ground-based observations}

We obtained $R$ - and $I$-band images of the field containing GRB 980703 using the Andalucía Faint Object
Table 1. The log of the observations

\begin{tabular}{lcll}
\hline \hline Telescope/Instrument & Filter & Date (UT) & Exposure Time (s) \\
\hline NOT/ALFOSC & $R$ & 1998 July 20 & $5 \times 600$ \\
& $I$ & & $5 \times 600$ \\
CAHA/CAFOS & $V$ & 2000 Jan. 5 & $1 \times 980$ \\
& & & $1 \times 1200$ \\
& $R$ & & $3 \times 900$ \\
HST/STIS & LP & 2000 June 12 & $4 \times 650$ \\
& & & $4 \times 666$ \\
& CL & 2000 June 18 & $1 \times 6$ \\
& & & $1 \times 615$ \\
& & $3 \times 613$ \\
& & $4 \times 666$ \\
\hline \hline
\end{tabular}

Spectrograph and Camera (ALFOSC) on the 2.56-meter Nordic Optical Telescope (NOT) at La Palma on 1998 July 20.172 (16.99 days after the burst). A log of all our observations is given in Table 1 . The instrumental gain was $1.0 \mathrm{e}^{-1} / \mathrm{ADU}$ and the read-out noise was $6 \mathrm{e}^{-1} /$ pixel. We calibrated the $R$-band data using six stars from Rhoads et al. (1998), and the $I$-band data using three stars from Vreeswijk et al. (1999). The magnitudes were measured in apertures with radii of 15 pixels $(=2 \prime \prime$. 82$)$. The derived relations between the instrumental and standard systems are $R=r+(8.440 \pm 0.011)+(-0.074 \pm 0.023)(r-i)$ and $I=i+(7.934 \pm 0.038)$. The $\mathrm{rms}$ of the residuals are 0.07 and $0.09 \mathrm{mag}$ respectively. We found combined host+OA magnitudes of $R=22.40 \pm 0.07$ and $I=21.71 \pm 0.09$ in an aperture of radius $1^{\prime \prime} 524\left(=8.5 h_{100}^{-1}\right.$ proper kpc) centred on the OA. The full-width at half-maxima $(F W H M \mathrm{~s})$ of the stellar profiles on the combined NOT image were 0.8 in the $R$ band, and $0^{\prime \prime} .9$ in the $I$ band.

In order to determine the magnitude of the host galaxy without contamination from the OA we obtained $V$ - and $R$-band images of the host galaxy with the Calar Alto Faint Object Spectrograph (CAFOS) at the Centro Astronómico Hispano-Alemán (CAHA) Observatory's 2.2-meter telescope on 2000 January 5 (551 days after the burst). These data have a gain of $2.3 \mathrm{e}^{-1} / \mathrm{ADU}$, a read-out noise of $5.06 \mathrm{e}^{-1} /$ pixel, and were calibrated using the same field stars and apertures as for the NOT data. The derived relations between the instrumental and standard systems are $V=v+(5.943 \pm 0.051)+(0.079 \pm 0.072)(v-r)$ and $R=r+(6.145 \pm 0.021)+(0.245 \pm 0.030)(v-r)$. The rms of the residuals in the calibrations are 0.031 and $0.027 \mathrm{mag}$ in $V$ and $R$ respectively. For the host galaxy we measured $V_{\text {gal }}=23.00 \pm 0.10$ and $R_{\text {gal }}=22.57 \pm 0.09$ in an aperture of radius 1 ". 524 . The CAHA combined $V$-band image had $F W H M=1$ 1.5 while the combined $R$-band image had $F W H M=1$.' 7 .

GRB 980703 is located at $\alpha=23: 59: 06.72 \delta=$ $+08: 35: 07.3$ (J2000) which correspond to Galactic coordinates of $\ell^{\mathrm{II}}=101.56, b^{\mathrm{II}}=-52^{\circ} .10$, hence the Galactic extinction is small. We have adopted a Galactic reddening of $E_{B-V}=0.058 \pm 0.020$ (corresponding to extinctions of $A_{V}=0.19 \pm 0.07, A_{R}=0.16 \pm 0.05$, and $\left.A_{I}=0.11 \pm 0.04\right)$ from the DIRBE/IRAF dust maps of 
Schlegel et al. (1998). The dereddened magnitudes of the host are $V_{0, \text { gal }}=22.81 \pm 0.12$ and $R_{0, \text { gal }}=22.41 \pm 0.10$.

If we assume that the host galaxy has a power law spectrum of the form $f \propto \nu^{\beta}$ then we derive $\beta_{\text {gal }}=$ $-1.08 \pm 0.59$, which gives $I_{0, \text { gal }}=21.91 \pm 0.23$.

\subsection{The HST data}

We used the HST/STIS to obtain CCD images of the host galaxy of GRB 980703 as part of the Cycle 9 proposal GO-8640. The total exposure times were 5178 seconds in the 50CCD (clear; hereafter referred to as CL) aperture and 5264 seconds in the F28X50LP (long pass; hereafter referred to as LP) aperture. The CL images were taken on 2000 June 12 (710 days after the burst) and the LP images were taken on 2000 June 18 (716 days after the burst). The CCD gain was set to $1 \mathrm{e}^{-} / \mathrm{ADU}$ and the read-out noise was $4.46 \mathrm{e}^{-} /$pixel. We used a four-point STIS-CCD-BOX dithering pattern with shifts of 2.5 pixels $(=0$ ". 127) between exposures. The data was pre-processed through the standard STIS pipeline and combined using the DITHER (v1.2) software (Fruchter \& Hook 2001) as implemented in $\operatorname{IRAF}^{1}(\mathrm{v} 2.11 .3) / \mathrm{STSDAS}(\mathrm{v} 2.1 .1)$. The "pixfrac" parameter was set to 0.6 , and we selected a final output scale of 0 "'0254/pixel. These observations are part of the Survey of the Host Galaxies of GRBs (http://www.ifa.au.dk/ hst/grb_hosts/index.html, Holland et al. 2000a).

We measured the total AB magnitude of the host galaxy on the CL and LP images in an aperture of radius 1".524. The zero points of Gardner et al. (2000) yield $\mathrm{AB}$ magnitudes of $22.87 \pm 0.02$ in the CL aperture and $22.50 \pm 0.03$ in the LP aperture.

\section{The host galaxy}

\subsection{The morphology of the host galaxy}

Figure 1a shows a contour plot of a section of the STIS CL image containing the host galaxy. The galaxy is extremely compact and has a somewhat irregular, "eggshaped" morphology. In order to quantitatively characterize the morphology of the galaxy we fitted elliptical isophotes to the CL and LP images of the host using Jedrzejewski's (1987) algorithm as implemented in the STSDAS task ISOPHOTE.ELLIPSE. These isophotes provided a resonable fit to the galaxy, but there are significant residuals as the isophotes are not elliptical. The mean observed ellipticity of the host on the CL image is $\epsilon=0.16 \pm 0.01$ for $0{ }^{\prime \prime} 05 \leq r \leq 0.51$, and the mean position angle of the semi-major axis on the CL image is $\theta_{0}=12.7 \pm 0.2$ east of north on the sky. We find $\epsilon=0.19 \pm 0.01$ and $\theta_{0}=9.2 \pm 0.2$ on the LP image. The variation of the ellipticity and position angle with radius is shown in Fig. 2. The host galaxy shows some evidence for

\footnotetext{
1 Image Reduction and Analysis Facility (IRAF), a software system distributed by the National Optical Astronomy Observatories (NOAO).
}

twisted isophotes as the position angle of the major axis rotates from $\approx+45^{\circ}$ to $\approx-45^{\circ}$ between 0.1 and $0^{\prime \prime} .5$ from the centre of the host galaxy. This change is seen in both the CL and LP images. These values have not been corrected for the effects of the point-spread function (PSF) on the observed shape of the galaxy. Schweizer (1979) and Holland (1998) have shown that the observed shape and surface brightness profile of a galaxy can be dominated by the effects of the PSF if the diameter of the galaxy is less than $\approx 8$ times the width of the PSF. The $F W H M$ of the STIS PSF in the CL and LP images is $\approx 00^{\prime \prime} 084$, so the shape of the PSF dominates the central regions of the host galaxy, and will effect the observed shape in the outer regions. The PSF has a mean ellipticity of 0.11 and a mean position angle of -31.5 in the CL image. This is $44^{\circ}$ from the observed major axis of the host galaxy, so the PSF will act to make the host appear to be less elliptical than it actually is.

In order to determine the intrinsic (i.e., before the light passed through the HST/STIS optics) surfacebrightness distribution of the host galaxy we fit twodimensional PSF-convolved generalized de Vaucouleurs profiles (Sersic 1968)

$S(R)=S_{\mathrm{e}} 10^{-b(n) / \ln (10)\left[\left(R / R_{\mathrm{e}}\right)^{1 / n}-1\right]}$

where $R_{\mathrm{e}}$ is the radius enclosing half the light from the galaxy and $S_{\mathrm{e}}$ is the surface brightness at $R_{\mathrm{e}}$, to the CL and LP images of the host galaxy using the software described in Holland et al. (1999). This software allows all of the model parameters to be fit simultaneously using a chi-square minimization scheme. The parameters of the best fitting models, along with the $\chi^{2}$ per degree of freedom (DOF) are listed in Table 2. The number of degrees of freedom in each fit is the number of pixels used in the fit minus the number of model parameters. The constant $b(n)$ is chosen so that $R_{\mathrm{e}}$ encloses half of the light of the galaxy. The mean surface brightness inside $R_{\mathrm{e}}$ is denoted by $\langle\mu\rangle_{\mathrm{e}}$. The formal 1- $\sigma$ errorbars for each parameter do not include contributions from correlations between the parameters, and may be affected by the systematic residuals caused by the presence of substructure in the host galaxy.

The standard de Vaucouleurs $R^{1 / 4}$ profile does not provide a good fit to the host galaxy, whereas a generalized de Vaucouleurs profile $\left(R^{1 / n}\right)$ with $n \approx 1$ provides a significantly better fit. However, both fits are formally strongly excluded by the data, which is due to the fact that there are large residuals in the central few pixels. Figure 1b shows the CL image of the host galaxy of GRB 980703 with the best-fitting two-dimensional PSFconvolved model subtracted. The model was computed to a radius of 64 pixels $\left(=1\right.$ '. $626=9.1 h_{100}^{-1}$ proper $\left.\mathrm{kpc}\right)$ from the centre of the galaxy. The model provides a good fit for $R \gtrsim 0^{\prime \prime} .25$, but there are systematic residuals in the inner $\approx 0$ '! 25 of the galaxy. However, we note that the model assumes that the galaxy has a smooth surface brightness distribution and does not include small-scale structure, 

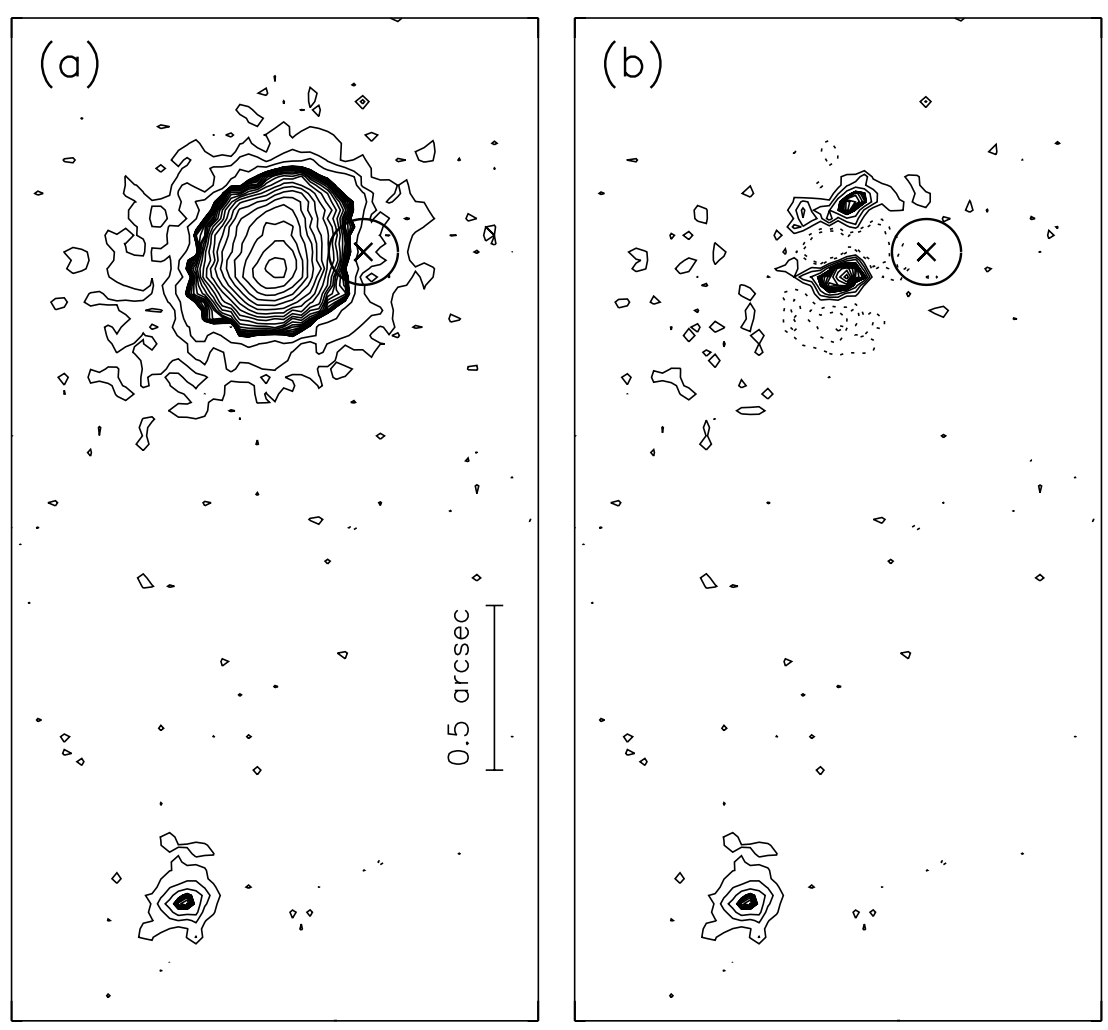

Fig. 1. Left panel: this figure shows a $1^{\prime \prime} 6 \times 33^{\prime \prime} 1$ section of the drizzled CL HST/STIS image. North is up and east is to the left. The lowest contours are shown linearly whereas the inner contours are logarithmic in order to better show the properties of the host over a large dynamic range. The "X" marks the location of GRB 980713 and the circle is our $1 \sigma$ error circle for the astrometric solution. The possible companion galaxy is located near the southern edge of the image. Right panel: this figure shows the host galaxy with the best-fitting $R^{1 / n}(n=1.07)$ model subtracted. The contour intervals are the same as for Fig. 1a. The systematic residuals seen near the centre of the host suggest that there is small-scale structure within the half-light radius. There is also an excess of light on the west side of the host

Table 2. The best-fitting two-dimensional PSF-convolved models to the CL and LP images of the host galaxy of GRB 980703

\begin{tabular}{ccc}
\hline \hline Parameter & $R^{1 / n}$ & $R^{1 / 4}$ \\
\hline CL Image & & \\
$R_{\mathrm{e}}$ & $0^{\prime \prime} 117 \pm 0^{\prime \prime} .001$ & $0^{\prime \prime} .188 \pm 0^{\prime \prime} .001$ \\
$\langle\mu\rangle_{\mathrm{e}}$ & $20.38 \pm 0.01$ & $21.06 \pm 0.01$ \\
$\epsilon$ & $0.26 \pm 0.01$ & $0.26 \pm 0.01$ \\
$\theta_{0}$ & $24^{\circ} 3 \pm 0.2$ & $21^{\circ} .9 \pm 0.1$ \\
$n$ & $1.07 \pm 0.01$ & 4 (fixed) \\
$\chi^{2} / \mathrm{DOF}$ & 11.253 & 13.050 \\
\hline \hline LP Image & & \\
$R_{\mathrm{e}}$ & $0^{\prime \prime} .134 \pm 0^{\prime \prime} .001$ & $0^{\prime \prime} .253 \pm 0^{\prime \prime} 001$ \\
$\langle\mu\rangle_{\mathrm{e}}$ & $20.27 \pm 0.01$ & $21.21 \pm 0.01$ \\
$\epsilon$ & $0.21 \pm 0.01$ & $0.17 \pm 0.01$ \\
$\theta_{0}$ & $10.4 \pm 0.5$ & $8.6 \pm 0.5$ \\
$n$ & $1.02 \pm 0.01$ & 4 (fixed) \\
$\chi^{2} / \mathrm{DOF}$ & 8.699 & 9.879 \\
\hline \hline
\end{tabular}

such as star-forming regions, within the galaxy. Therefore, we do not expect to obtain $\chi^{2} / \mathrm{DOF} \approx 1$ for our fits. The best-fitting model provides a good fit over most of the host galaxy except for the central few pixels, and the observed residuals are consistent with the presence of substructure in the central regions of the galaxy.

A Sersic (1968) model with $n=1$ corresponds to an exponential disk. Therefore, our modelling suggests that the host galaxy of GRB 980703 has a disk-like structure, with smaller sub-structure in the central regions. The excess light seen on the east side of the galaxy after subtracting the best-fitting model (see Fig. 1b), may be a faint spiral arm. The fitted half-light radii of our Sersic (1968) models correspond to disk scale radii of $00^{\prime \prime} 196 \pm 0$ "'002 on the CL image and $0.225 \pm 0$. 002 in LP.

The derived half-light radius $\left(\approx 0.72 h_{100}^{-1}\right.$ proper $\left.\mathrm{kpc}\right)$ is much smaller than the half-light radii seen in local late and early type galaxies (Im et al. 1995). Phillips et al. (1997) and Guzmán et al. (1997) studied galaxies in the Hubble Deep Field North that were selected to be compact (i.e., had measured half-light radii smaller than 0.5 including the effect of the WFPC2 PSF). Their 


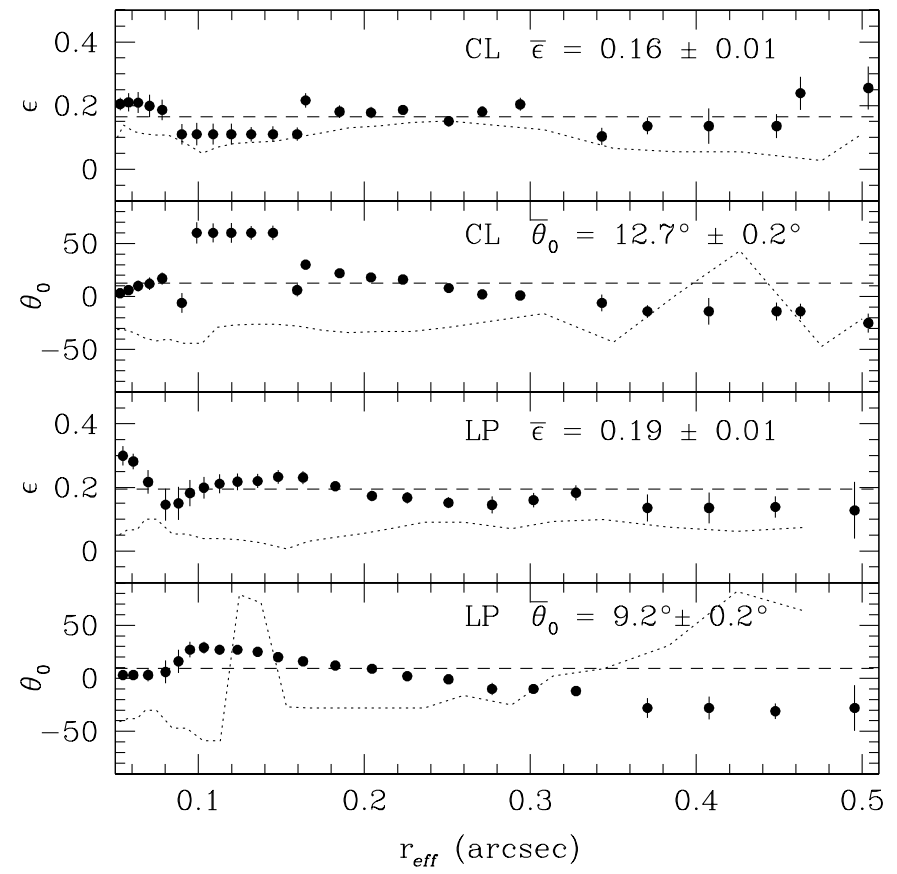

Fig. 2. The upper two panels show the observed ellipticity and orientation profiles for the host galaxy of GRB 980703 in the CL aperture (filled circles). The dashed line is the weighted mean of the ellipticity (or position angle) and the dotted line is the profile of the PSF. The data points near $0 . \prime 1$ are constant because the fitting algorithm was unable to converge at these radii. The derived position angles are not reliable for $\epsilon \approx 0$. The lower two panels show the same thing in the LP aperture. The data has not been corrected for the effects of the PSF

galaxies covered a range of redshifts from $z \approx 0.4$ to $z \approx 1.0$ and appear to have properties very similar to the host galaxy of GRB 980703, namely $V-I$ colours in the range $0.7<V-I<1.1$, half-light radii in the range $0.65 h_{100}^{-1} \leq R_{\mathrm{e}} \leq 2.6 h_{100}^{-1} \mathrm{kpc}$ (converted to our cosmology and not corrected for the effect of the WFPC2 PSF), and observed $I$-band magnitudes in the range $21 \leq I \leq 24$ for the galaxies at redshifts similar to the redshift of GRB 980703. The spectra of these galaxies are similar to the spectrum of the host galaxy-i.e., dominated by strong emission lines from O II, and O III, as well as Balmer lines (Guzmán et al. 1997; Djorgovski et al. 1998a). The restframe equivalent width $(E W)$ of the O II line from the host galaxy was found by Djorgovski et al. (1998a) to be $46 \AA$, which is in the middle of the range $5 \leq E W \leq 94 \AA$ found by Guzmán et al. (1997) for the compact galaxies in the Hubble Deep Field North.

\subsection{The colour profile of the host galaxy}

The host galaxy of GRB 980703 has $V_{0}=22.81 \pm 0.12$ and $(V-R)_{0}=0.40 \pm 0.15$ measured in an aperture with a radius of $1^{\prime \prime} .524$ on the CAHA images. This colour is consistent with the colours predicted by Bloom et al. (1998) and Vreeswijk et al. (1999) based on fitting a single power law plus a constant flux to the optical decay of the OA. We find no evidence for azimuthal variations in colour,

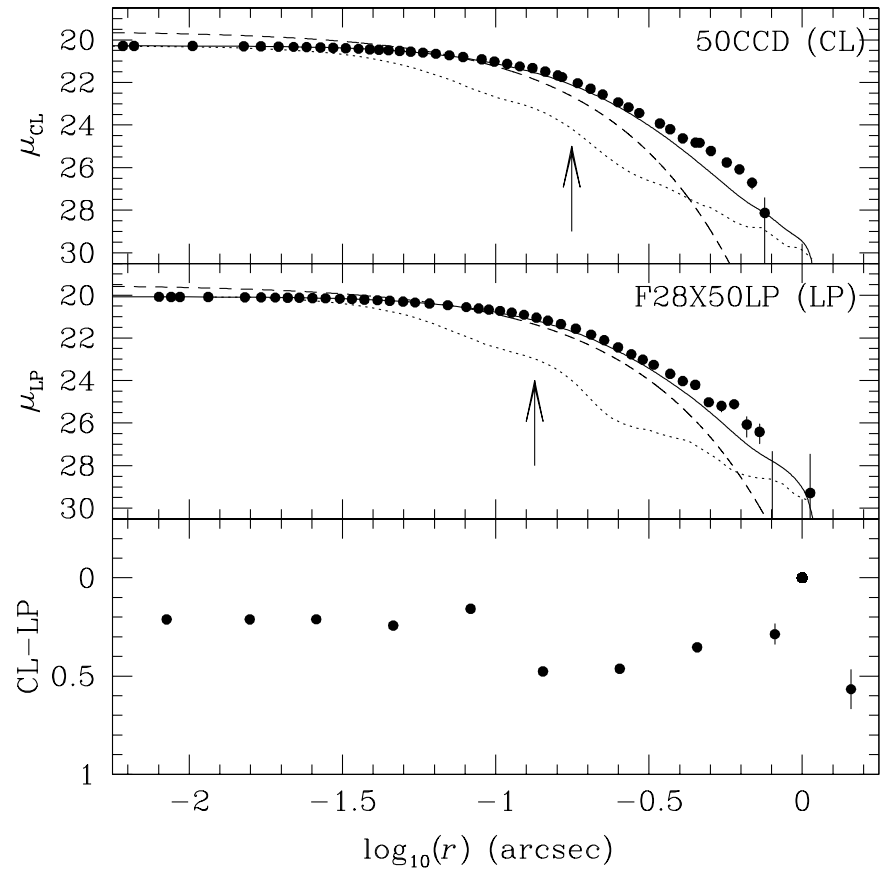

Fig. 3. The top panel shows the azimuthally average surface brightness profile that we derived from the HST /STIS CL image of GRB 980703 (filled circles). The solid line is the best fitting PSF-convolved $r^{1 / n}$ profile. The dashed line shows the best-fitting model after correcting for the PSF, and the dotted line shows the point spread function for the image. The arrow indicates the half-light radius of the unconvolved model. The middle panel shows the same information for the LP band data. The lower panel shows the azimuthally averaged colour gradient in the host. The data has not been corrected for Galactic extinction or reddening

but the center of the galaxy is $\approx 0.2$ mag bluer than the outer regions of the galaxy with the step in the colour occurs at approximately the effective radius of the bestfitting model (see Sect. 3.1). Matthews \& Gallacher (1997) note that this sort of trend in colour is unique to late-type disc galaxies and some dwarf galaxies. Figure 3 shows the surface brightness profiles and the azimuthally averaged colour gradient that were derived from the STIS CL and LP images.

\subsection{Star formation in the host galaxy}

At a redshift of $z=0.9661$ a rest-frame wavelength of $2800 \AA$ corresponds to the observer's $V$ band. Therefore, we are able to use Eq. (2) of Madau et al. (1998) to estimate the star formation rate in the host galaxy from the continuum flux at $2800 \AA$. For a Salpeter (1955) initial mass function we find a starformation rate of $(4.1 \pm 0.5) h_{100}^{-2} \quad \mathcal{M}_{\odot} \quad \mathrm{yr}^{-1}$ while a Scalo (1986) initial mass function yields a star-formation rate of $(6.4 \pm 0.7) h_{100}^{-2} \mathcal{M}_{\odot} \mathrm{yr}^{-1}$. If we take $h_{100}=0.7$ (de Bernardis et al. 2000) then the star-formation rate is $\approx 8-13 \mathcal{M}_{\odot} \mathrm{yr}^{-1}$, depending on the assumed initial mass function. However, this calculation assumes that there is no extinction in the host galaxy, which is probably an 
incorrect assumption. If $A_{V}=0.3$ in the host then the star-formation rate becomes $\approx 10-20 \mathcal{M}_{\odot} \mathrm{yr}^{-1}$, while if if $A_{V}=2.2$ in the host then the star-formation rate is $\approx 65$ $100 \mathcal{M}_{\odot} \mathrm{yr}^{-1}$. These values are consistent with the starformation rate obtained by Djorgovski et al. (1998a) using the strength of the $[\mathrm{O}$ II $]$ emission line $\left(\approx 10 \mathcal{M}_{\odot} \mathrm{yr}^{-1}\right.$ with $\left.A_{V}=0.3 \pm 0.3\right)$.

\subsection{The luminosity of the host galaxy}

Lilly et al. (1995) find a typical magnitude of $\left(M_{B}^{*}\right)_{\mathrm{AB}}=$ -21.4 for blue galaxies at $0.75 \leq z<1.00$, if $\left(H_{0}, \Omega_{m}, \Omega_{\Lambda}\right)=(50,1,0)$. For our adopted cosmology, this corresponds to $\left(M_{B}^{*}\right)_{\mathrm{AB}}=-21.47+5 \log _{10}\left(h_{100}\right)$. At $z=0.9661$ the observed Kron-Cousins $I$ band is approximately half-way between the rest-frame Johnson $U$ and $B$ bands. If we assume that the host galaxy has a power-law spectrum with $\beta_{\text {gal }}=-1.08 \pm 0.59$ (from the CAHA data, see Sect. 2.1) then $L_{B} \approx(3.2 \pm 0.7) h_{100}^{2} L_{B}^{*}$ where $L_{B}^{*}$ is the rest-frame $B$-band luminosity of a typical blue galaxy at $z=0.9961$. Most of the uncertainty in the luminosity comes from the uncertainty in the shape of the host galaxy's rest-frame spectrum at $\lambda \lesssim 4000 \AA$. For $h_{100}=$ 0.7 the rest-frame $B$-band luminosity is $(1.6 \pm 0.4) L_{B}^{*}$. Therefore, the host is approximately an $L^{*}$ galaxy with a total luminosity similar to that of other galaxies at $z=0.9661$. Our comparison of the total $B$-band luminosity of the host to $L_{B}^{*}$ is, however, somewhat uncertain because $M^{*}$ is highly correlated with the slope of the faint end of the galaxy luminosity function, and with its normalisation, $\phi^{*}$ (Lilly et al. 1995).

The specific star-formation rate per unit blue luminosity of the host galaxy of GRB 980703, if we ignore extinction within the host, is $\approx 6.5 \mathcal{M}_{\odot} \mathrm{yr}^{-1} L_{B}^{*-1}$, which is similar to that of other GRB host galaxies. The specific star-formation rate is $\approx 0.6$ times that of the hosts of GRB $970508\left(11 \mathcal{M}_{\odot} \mathrm{yr}^{-1} L_{B}^{*-1}\right)$ and GRB 990123 $\left(11 \mathcal{M}_{\odot} \mathrm{yr}^{-1} L_{B}^{*-1}\right)$ and $\approx 1.5$ times that of the host of GRB $990712\left(4.4 \mathcal{M}_{\odot} \mathrm{yr}^{-1} L_{B}^{*}{ }^{-1}\right)$. If the extinction in the host is non-negligible then the true star-formation rate per unit blue luminosity may be much higher.

There is a faint $(\mathrm{CL}=27.14 \pm 0.05, \mathrm{LP}=26.29 \pm 0.06)$ object $2^{\prime \prime}\left(=11.2 h_{100}^{-1}\right.$ proper $\left.\mathrm{kpc}\right)$ south of the host galaxy (see Fig. 1a), which may be a companion galaxy. This object has $F W H M=00^{\prime \prime} 097$, which is slightly larger than the resolution of the CL image $(0.084)$ and appears to have a faint extended structure around it.

\subsection{The position of GRB 980703 in the host galaxy}

The paucity of stars in the STIS images makes it difficult to accurately determine the location of GRB 980703 within the host galaxy. Only four stars are present in both the STIS CL image and our ground-based images, and two of these are very faint in our ground based images. We used these four stars to determined the location of the
OA relative to the galaxy by comparing the STIS CL image to an $R$-band image obtained with the NOT on 1998 July 7.163 (Pedersen et al. 1998). At the epoch of the NOT observation, the OA contributed $\approx 25 \%$ of the total host+OA flux, so we registered, scaled, and smoothed the STIS image to match the seeing and intensity of the NOT image, then subtracted the STIS image from the NOT image. The OA is found to be offset $0 . .23$ west and 0. . 02 north of the center of the host galaxy with an estimated $1 \sigma$ error of approximately \pm 0 !' 10 . This is within approximately $1 \sigma$ of the location determined by Bloom et al. (2001). We find the GRB to have occurred $00^{\prime \prime} 23 \pm 0$ " 14 from the centre of the host at a position angle of $-85.0 \pm 4.6$, while Bloom et al. (2001) find that the GRB occurred at a distance of $0^{\prime \prime} .11 \pm 0.09$ with a position angle of $-28^{\circ} .9 \pm 0.3$. The $1 \sigma$ error ellipses of these two position determinations overlap, but we note that Bloom et al. (2001) used deep Keck images to obtain an astrometric solution involving 23 objects while our solution involved only four stars.

\section{The late-time light-curve of the optical afterglow}

Figures 4 and 5 shows the $R$ - and $I$-band light curves for the OA based on measurements taken from the literature and on our own NOT measurements. The magnitudes have been corrected for the contribution from the underlying host galaxy and for Galactic extinction. The light from the host galaxy was removed by subtracting the total light from the host galaxy as determined from our CAHA images.

We fit both a single power law $\left(f_{\nu}(t)=f_{\nu}(0) t^{\alpha}\right)$, where $f_{\nu}(t)$ is the flux in $\mu$ Jy $t$ days after the burst, and a broken power law

$f_{\nu}(t)=\left\{\begin{array}{l}f_{\nu}\left(t_{\mathrm{b}}\right)\left(t / t_{\mathrm{b}}\right)^{\alpha_{1}}, \text { if } t \leq t_{\mathrm{b}} \\ f_{\nu}\left(t_{\mathrm{b}}\right)\left(t / t_{\mathrm{b}}\right)^{\alpha_{2}}, \text { if } t>t_{\mathrm{b}}\end{array}\right.$

where $\alpha_{1}$ is the slope before the break, $\alpha_{2}$ is the slope after the break, and $t_{\mathrm{b}}$ is the time in days after the burst that the break occurred, to the $R$-band data as described in Holland et al. (2000) and Jensen et al. (2001). Only data $t \leq 10$ days after the burst were used for the fit since a type Ic supernova (SN) like SN1998bw would not make a significant contribution to the total flux at these times. The parameters of our best fits are listed in Table 3 and the fits themselves are shown in Figs. 4 and 5. The small $\chi^{2} / \mathrm{DOF}$ values for both the single and broken power law fits reflect the large formal uncertainties in the late time data that arise from subtracting the OA from the host at a time when both the OA and the host had similar magnitudes. The small number of data points makes it impossible to fit a broken power law in the $I$-band, so we scaled the broken power law that was fit to the $R$-band data to match (in a chi-square sense) the early $I$-band photometry. The best fit occurred for $I_{0}\left(t_{\mathrm{b}}\right)=20.77 \pm$ 0.05 . The broken power law is formally a slightly better fit to the $R$-band light curve than a single power law is, 


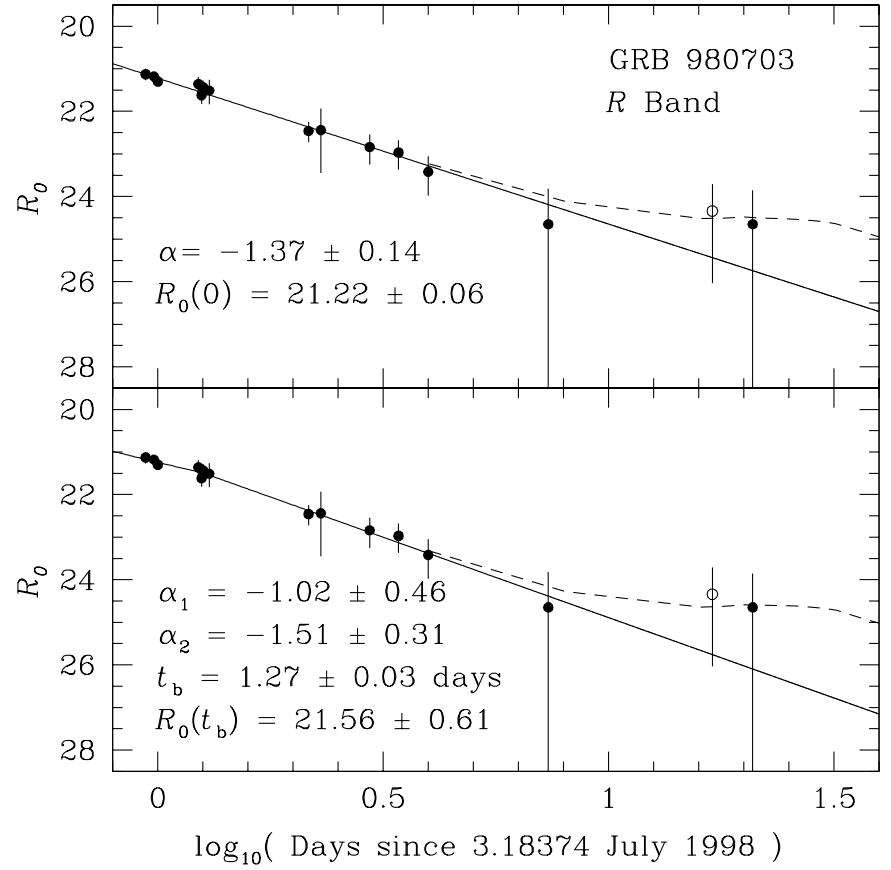

Fig. 4. The upper panel shows the $R$-band photometry for the OA associated with GRB 980703 (filled circles) after subtracting the light from the host galaxy $\left(R_{\text {gal }}=22.57 \pm 0.09\right)$ and correcting for extinction in the Milky Way $\left(A_{R}=0.16 \pm 0.05\right)$. The open circle shows our NOT data point. The solid line is the best-fitting broken single law to all the data. The dashed line is the predicted $R$-band magnitude for a type Ic SN like SN1998bw at a redshift of $z=0.9661$. The lower panel shows the same photometry with the best-fitting broken power law and the corresponding prediction with an SN Ic like SN1998bw included

although an F-test suggests that the broken power law is only significant at about the $1 \sigma$ level. There are several gaps in the decay curve, and large error bars in the latetime data due to the subtracting of the light from the bright host galaxy, so we cannot unambiguously conclude that there is a break in the light curve. Therefore, we prefer the single power law fit since it requires fewer free parameters to fit the light curve. We note, however, that breaks have been seen in most well-sampled GRB light curves, and that the time and magnitude of the break for GRB 980703 is consistent with the breaks seen for several other OAs.

There is growing evidence that at least some GRBs are associated with supernovae. The prototype for this association is the temporal and positional coincidence of GRB 980425 and the unusual type Ib/c SN1998bw (Galama et al. 1998). VLT and HST/STIS images show that this GRB/SN occurred in a star-forming region in the sub-luminous barred spiral galaxy ESO 184-G82 (Sollerman et al. 2000; Fynbo et al. 2000). Evidence for a GRB/SN association has also been seen for GRB 980326 (Castro-Tirado \& Gorosabel 1999; Bloom et al. 1999), GRB 970228 (Dar 1999; Reichart 1999; Galama et al. 2000), GRB 970514 (Germany et al. 2000), GRB 990712 (Fruchter et al. 2000; Björnsson et al. 2001), and

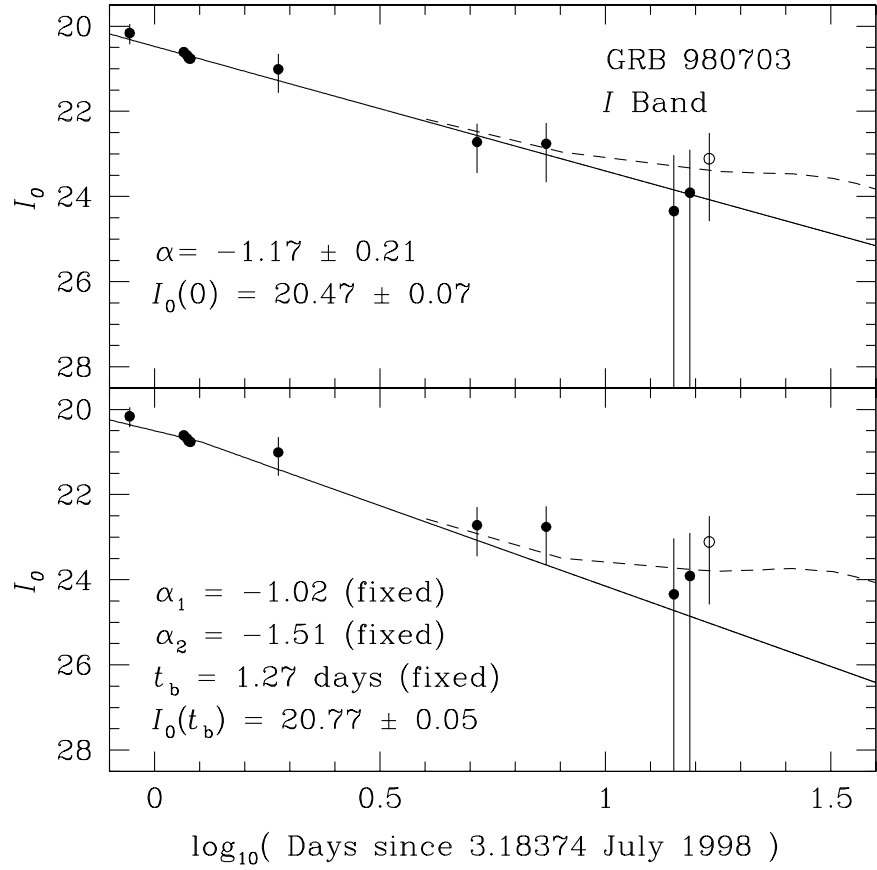

Fig. 5. The upper panel shows the $I$-band photometry for the OA associated with GRB 980703 (filled circles) after subtracting the light from the host galaxy $\left(I_{\text {gal }}=22.02 \pm 0.23\right)$ and correcting for extinction in the Milky Way $\left(A_{I}=0.11 \pm 0.04\right)$. The open circle shows our NOT data point. The solid line is the best-fitting single power law to all the data. The dashed line is the predicted $I$-band magnitude for a type Ic SN like SN1998bw at a redshift of $z=0.9661$. The lower panel shows the same photometry with the best-fitting broken power law and the corresponding prediction for an SN Ic like SN1998bw. These data should be considered less reliable than the $R$ band data since $I_{\text {gal }}$ was not measured directly, but determined by assuming that the host has a power-law spectrum with $\beta_{\text {gal }}=-1.08 \pm 0.59$ (see Sect. 2.1)

GRB 991002 (Terlevich et al. 1999). In addition, Hudec et al. (1999) have identified five more candidates for SN/GRB associations based on positional and temporal coincidence. In Figs. 4 and 5 we show the predicted light curves for GRB 980703 if it was associated with a type Ic SN like SN1998bw. Although both the $R$ - and $I$-band data are consistent with both a pure power law decline and a broken power law decline the addition of an SN Ic like SN1998bw results in a better fit to the data. However, the large error bars in the late-time data prevent us from being able to reliably distinguish between a SN and a no-SN scenario for GRB 980703.

It has recently been suggested that a dust echo could produce an excess flux in the decay of the late-time optical light curve of a GRB similar to the flux excesses seen in GRB 970228 and GRB 980326 (Esin \& Blandford 2000). Using their method we predict an excess $R$-band flux due to dust 17 days after the burst of $F_{\nu_{\mathrm{ob}}}^{\mathrm{E}} \approx 0.03 \mu \mathrm{Jy}$, approximately $2 \sigma$ less than the observed excess flux at this time, assuming a single power law decay. Therefore, we are unable to distinguish between the dust echo and no dust echo cases. 
Table 3. The parameters of the best-fitting single power law and broken power law to the decay of the optical light curve of GRB 980703

\begin{tabular}{ccc}
\hline \hline & $R$ & $I$ \\
\hline Single Power Law & & \\
$\alpha$ & $-1.37 \pm 0.14$ & $-1.17 \pm 0.21$ \\
$f_{0}(\mu \mathrm{Jy})$ & $9.805 \pm 0.512$ & $15.373 \pm 0.930$ \\
$\operatorname{mag}_{0}(0)$ & $21.22 \pm 0.06$ & $20.47 \pm 0.07$ \\
$\chi^{2} / \mathrm{DOF}$ & 0.268 & 0.322 \\
DOF & 11 & 6 \\
\hline Broken Power Law & & \\
$\alpha_{1}$ & $-1.02 \pm 0.46$ & -1.02 (fixed) \\
$\alpha_{2}$ & $-1.51 \pm 0.31$ & -1.51 (fixed) \\
$t_{\mathrm{b}}($ days $)$ & $1.27 \pm 0.03$ & 1.27 (fixed) \\
$f\left(t_{\mathrm{b}}\right)(\mu \mathrm{Jy})$ & $7.541 \pm 0.061$ & $11.750 \pm 0.584$ \\
$\operatorname{mag}_{0}\left(t_{\mathrm{b}}\right)$ & $21.51 \pm 0.61$ & $20.77 \pm 0.05$ \\
$\chi^{2} / \mathrm{DOF}$ & 0.231 & 0.496 \\
$\mathrm{DOF}$ & 9 & 7 \\
\hline \hline
\end{tabular}

The $R$-band slope of the best-fitting single power law is $\alpha=-1.37 \pm 0.14$, which is consistent with other values in the literature. Combining the optical slope with the observed slope of the $X$-ray decay $\left(\alpha_{\mathrm{X}}<-0.91\right.$, Vreeswijk et al. 1999), and assuming that the GRB is a collimated outflow into a region with a $\rho(r) \propto r^{-\delta}$ density profile (e.g. Panaitescu et al. 1998; Mészáros et al. 1998), implies that $\delta=2$ (Panaitescu \& Kumar 2001). This suggests that the fireball is expanding into a local medium dominated by a pre-existing stellar wind, similar to the situation that Jaunsen et al. (2001) found for GRB 980519. The optical spectrum of the early OA (1.2 days after the burst) has a slope of $\beta_{\mathrm{OA}}=-2.71 \pm 0.12$ (Vreeswijk et al. 1999). For an expansion into a pre-existing stellar wind the spectral index of the OA is given by $\beta_{\mathrm{OA}}=(1+2 \alpha) / 3=-0.58 \pm 0.09$, which is inconsistent with the observed early-time slope of OA's optical spectrum. This discrepancy, however, can be resolved if there are several magnitudes of extinction along the line of sight to the GRB. The extinction measurements in Sect. 1 are consistent with $A_{V}$ decreasing by $\approx 0.3 \mathrm{mag} /$ day from $A_{V} \approx 1.5$ one day after the burst to $A_{V} \approx 0.5$ five days after the burst. An examination of the spectrum of Djorgovski et al. (1998b) suggests that $\beta_{\mathrm{OA}} \approx-1.6 \pm 0.2$ in the $R$ band 4.4 days after the burst, which suggests that the burst evolved to become bluer at later times, which is consistent with $A_{V}$ decreasing with time. This suggests that some of the discrepancy between the observed spectral index and the spectral index which is predicted from the decay of the optical and $X$-ray light curves may be due to the time evolution of the OA's spectrum that result from changes in the amount of dust along the line of sight to the burst during the first few days after the burst.

\section{Conclusions}

The host galaxy of GRB 980703 is a compact blue, starforming galaxy with $V_{\text {gal }}=23.00 \pm 0.10$ and $(V-R)_{\text {gal }}=$ $0.43 \pm 0.13$. The central regions of the host are $\approx 0.2 \mathrm{mag}$ bluer than the outer regions. Its rest-frame $B$-band luminosity is $L_{B} \approx 3.2 h_{100}^{2} L_{B}^{*}$, and it has a star-formation rate, based on the integrated flux at $2800 \AA$, of $8-13 \mathcal{M}_{\odot} \mathrm{yr}^{-1}$, assuming no extinction in the host. This suggests that the host galaxy is undergoing a phase of active star formation similar to what has been seen in other GRB host galaxies. We find that the galaxy is best fit by a Sersic (1968) $R^{1 / n}$ profile with $n \approx 1$ and a half-light radius of 0.13 (= $0.72 h_{100}^{-1}$ proper kpc). This corresponds to an exponential disk with a scale radius of $0^{\prime \prime} .22\left(=1.21 h_{100}^{-1}\right.$ proper $\left.\mathrm{kpc}\right)$. The residuals of the fit show that the morphology of the host is irregular, but we are unable to determine if the location of GRB 980703 coincided with any special features in the host. The galaxy resembles the compact star-forming galaxies that Phillips et al. (1997) and Guzmán et al. (1997) studied galaxies in the Hubble Deep Field North.

The optical decay of GRB 980703 followed the same broad pattern established by other long-duration GRBs: a power-law decay, perhaps with a break a few days after the burst, possibly followed by an increase in flux $\approx 10(1+z)$ days after the burst.

Acknowledgements. This work was supported by the Danish Natural Science Research Council (SNF). JG acknowledges the receipt of a Marie Curie Research Grant from the European Commission. MA acknowledges the Astrophysics group of the Physics Department of University of Oulu for support of his work. GB acknowledges partial support from the Icelandic Research Council and the University of Iceland Research Fund. We would also like to thank the anonymous referee for a careful reading of the original manuscript, and for useful comments that greatly improved this paper.

\section{References}

Björnsson, G., et al. 2001, ApJ, submitted

Bloom, J. S., Frail, D. A., Kulkarni, S. R., et al. 1998, ApJL, 508, L21

Bloom, J. S., Kulkarni, S. R., Djorgovski, S. G., \& Eichelberger, A. C. 1999, Nature, 401, 452

Bloom, J. S., Kulkarni, S. R., \& Djorgovski, S. G. 2001, AJ, submitted [astro-ph/0010176]

Castro-Tirado, A. J., \& Gorosabel, J. 1999, A\&AS, 138, 449

Castro-Tirado, A. J., Zapatero-Osorio, M. R., Gorosabel, J., et al. 1999, ApJL, 511, L85

Dar, A. 1999, GCNC, 346

de Bernardis, P., et al. 2000, Nature, 404, 955

Djorgovski, S. G., Kulkarni, S. R., Bloom, J. S., et al. 1998a, ApJL, 508, L17

Djorgovski, S. G., Kulkarni, S. R., Goodrich, R., Frail, D. A., \& Bloom, J. S. 1998b, GCNC, 139

Esin, A. A., \& Blandford, R. 2000, ApJL, 534, L151

Frail, D. A., Halpern, J. P., Bloom, J. S., Kulkarni, S. R., \& Djorgovski, S. G. 1998, GCNC, 128

Fruchter, A. S., \& Hook, R. N. 2001, PASP, submitted [astro-ph/9808087]

Fruchter, A. S., Vreeswijk, P., Hook, R., \& Pian, E. 2000, GCNC, 752

Fynbo, J. U., Holland, S., Andersen, M. I., et al. 2000, ApJL, 542, L89 
Galama, T. J., Vreeswijk, P. M., van Paradijs, J., et al. 1998, Nature, 395, 670

Galama, T. J., Tanvir, N., Vreeswijk, P. M., et al. 2000, ApJ, 536,185

Gardner, J. P., Baum, S. A., Brown, T. M., et al. 2000, AJ, 119,486

Germany, L. M., Reiss, D. J., Sadler, E. M., Schmidt, B. P., \& Stubbs, C. W. 2000, ApJ, 533, 320

Guzmán, R., Gallego, J., Koo, D. C., et al. 1997, ApJ, 489, 559

Holland, S. 1998, PASP, 110, 759

Holland, S., Côté, P., \& Hesser, J. E. 1999, A\&A, 348, 418

Holland, S., Fynbo, J. U., Thomsen, B., et al. 2000a, GCNC, 698

Holland, S., Fynbo, J. U., Thomsen, B., et al. 2000b, GCNC, 715

Holland, S., Björnsson, G., Hjorth, J., \& Thomsen, B. 2000, A\&A, 364, 467

Hudec, R., Hudcová, V., \& Hroch, F. 1999, A\&AS, 138, 475

Im, M., Casertano, S., Griffiths, R. E., Ratnatunga, K. U., \& Tyson, J. A. 1995, ApJ, 441, 494

Jaunsen, A. O., Hjorth, J., Björnsson, G., et al. 2001, ApJ, 546,127

Jedrzejewski, R. I. 1987, MNRAS, 226, 747

Jensen, B. L., Fynbo, J. U., Gorosabel, J., et al. 2001, A\&A, submitted [astro-ph/0005609]

Levine, A., Morgan, E., \& Muno, M. 1998, IAUC, 6966

Lilly, S. J., Tress, L., Hammer, F., Crampton, D., \& Le Fèvre, O. 1995, ApJ, 455, 108

Madau, P., Pozzetti, L., \& Dickinson, M. 1998, ApJ, 498, 106
Matthews, L. D., \& Gallagher III, J. S. 1997, AJ, 114, 1899

Mészáros, P., Rees, M. J., \& Wijers, R. A. M. J. 1998, ApJ, 499, 301

Panaitescu, A., \& Kumar, P. 2001, ApJ, submitted [astro-ph/0010257]

Panaitescu, A., Mészáros, P., \& Rees, M. J. 1998, ApJ, 503314

Pedersen, H., Korhonen, H., \& Jaunsen, A. O. 1998, GCNC, 142

Phillips, A. C., Guzmán, R., Gallego, J., et al. 1997, ApJ, 489, 543

Reichart, D. E. 1999, ApJL, 494, L111

Rhoads, J., Downes, R., \& Christensen, J. 1998, GCNC, 144

Salpeter, E. E. 1955, ApJ, 121, 161

Scalo, J. N. 1986, Fund. Cosm. Phys., 11, 1

Schlegel, D. J., Finkbeiner, D. P., \& Davis, M. 1998, ApJ, 500, 525

Schweizer, F. 1979, ApJ, 233, 23

Sersic, J. L. 1968, Atlas de galaxias astrales, Observatorio Astronomico, Cordoba

Sokolov, V. V., Fatkhullin, T. A., \& Komarova, V. N. 2001 [astro-ph/0001357]

Sollerman, J., Kozma, C., Fransson, C., et al. 2000, ApJL, 537 , L127

Terlevich, R., Fabian, A., \& Turatto, M. 1999, IAUC, 7269

Vreeswijk, P. M., Galama, T. J., Owens, A., et al. 1999, ApJ, 523,171

Zapatero Ostorio, M. R., Castro-Tirado, A. J., \& Gorosabel, J. 1998, IAUC, 6967 